\title{
Development of optical detection for antibiotic residues: oxytetracycline in freshwater aquaculture
}

\author{
Merryl Jean Marquez ${ }^{1}$, Ciara Jane Roncales $^{1}$, Roma Ayanah Tigcal ${ }^{1}$, and Edna Quinto ${ }^{1,2, *}$ \\ ${ }^{1}$ Chemical Engineering Department, Faculty of Engineering, University of Santo Tomas \\ ${ }^{2}$ Research Center for the Natural and Applied Science, University of Santo Tomas, España Boulevard Manila 1015, \\ Philippines
}

\begin{abstract}
This study satisfies the primary need for a simple monitoring scheme for antibiotic residue content in freshwater systems to regulate and prevent immunization of aquaculture species to antibiotics. A series of antibiotic solutions with decreasing concentrations (from $5 \mathrm{ppm}$ to $1 \mathrm{ppm}$ ) were prepared, with each solution containing fixed amounts of the reagents ( $\mathrm{p}$-Chloranilic acid in acetonitrile solution, and pure acetonitrile) and a buffer solution to distinctly simulate the environmental conditions of actual freshwater systems. Using the UV-Vis Spectrophotometer with the optimum wavelength of $519 \mathrm{~nm}$, the stability, repeatability, and sensitivity tests were performed, where relative standard deviation (RSD) values of less than $10 \%$ were obtained. The limit of detection was also calculated to be $0.54 \mathrm{ppm}$, using the RSD result from the sensitivity test. Actual freshwater sample was gathered and subjected to a repeatability test using the same working wavelength. Results showed that the trend in the absorbance reading for the real water sample differed with the laboratory standard samples. This shift may have been caused by other interfering chemicals used in aquaculture that were present in the sample. Modified detection method of Oxytetracycline (OTC) in freshwater was made to provide solution to the main objective.
\end{abstract}

\section{Introduction}

Dubbed as the "Center of Marine Biodiversity" in the world, Philippines is known for its sanctified waters, providing home to millions of aquatic species, some of which are only found here in the country. This interesting fact paved way for the growth and development of the fisheries industry in the country. However, during the past few years, the increased fish consumption and the worldwide exportation of marine species from the Philippine reefs drove the expansion of the aquaculture industry [1]. Aquaculture, as defined by the National Oceanic and Atmospheric Administration, involves the breeding, rearing, and harvesting of fishes and other aquatic species in marine and freshwater environments [2].

According to the 2014-2016 annual Fisheries Statistics of the Philippines, under the Philippine Statistics Authority, the aquaculture industry provided the highest volume of production among the fisheries sectors, comprising about $40 \%$ of the total volume of fish production in the country - roughly amounting to 2.3 million MT of fishes being produced on average from 2014-2016.

One of the major problems experienced in the aquaculture industry is disease contraction among aquatic species. Infectious diseases provide continuing hazard towards major stock losses, arising from increasing death rate among the species being bred. As a solution, many pond owners resort to using antibiotics to treat these diseases [3].

Antibiotics are widely used in human and veterinary medicines, in agriculture, and in aquaculture as growth promoters [4]. This has become a necessity due to its various impacts in ecosystem functioning, as well as in public health. However, contamination of veterinary drugs in aquatic environment has become an increasingly important and alarming issue since these residues remotely increase the population of drugresistant bacteria and further promote antibioticresistance infection to aquaculture species [1].

To minimize the risk in both aquaculture environment setting and human health, several determination methods, such as UV-Vis spectrophotometer, immuno-chromatography and highperformance liquid chromatography, are used to closely monitor the concentrations of antibiotic residues in water. However, these methods are proved to be timeconsuming.

The general objective of this study is to provide the aquaculture industry a method for antibiotic concentration determination.

Specifically, the objectives of the study are:

(1) To determine the working wavelength of the testing method;

(2) to determine the accuracy, reliability and the limit of detection of the testing method to be used;

(3) to partially determine the concentration of Oxytetracycline in freshwater; and

* Corresponding author: ecquinto@ust.edu.ph 
(4) to produce a time-efficient method of determining Oxytetracycline residue concentration in freshwater.

\section{Materials and methods}

\subsection{The equipment used}

The equipment used in the experimentation was UV-Vis Spectrophotometer Shimadzu Model-2600. The UV-Vis Spectrophotometer was readily available in the Analytical Balance Room of the Chemical Engineering Department located at the Roque Ruaño building of University of Santo Tomas.

\subsection{Preparation of cocktail solution}

A cocktail solution, consisting of pure Acetonitrile and $0.2 \%$ Chloranilic Acid-Acetonitrile solution, was prepared. When mixed with Oxytetracycline (OTC) dissolved in distilled water, and $\mathrm{pH} 9$ buffer to control the $\mathrm{pH}$ of the solution and to simulate the real water environment [7], the cocktail solution reacts with OTC through charge transfer complexation. The cocktail solution was also mixed with the freshwater sample that was tested for OTC concentration.

\subsection{Determination of working wavelength}

To be able to determine the working wavelength, UVVis Spectrophotometer was used, and a wavelength scan was done from $700 \mathrm{~nm}$ to $350 \mathrm{~nm}$, first for cocktail solution, then a cocktail solution with OTC. The difference between the two was calculated to determine the optimum wavelength. A plot of the absorbance vs wavelength was done for the two and their difference, the optimum wavelength was determined by spotting the highest difference in absorbance.

\subsection{Stability}

At the determined working wavelength in II 3 , the stability of the cocktail was measured by reading the absorbance for one hour. The results were plotted, and the relative standard deviation was calculated based on the results.

\subsection{Repeatability}

Repeatability was tested by reading the absorbance at the same working wavelength with a total time of 8 minutes per trial, 4 minutes for cocktail and another 4 minutes for cocktail with OTC and buffer. In this part, concentration of $3 \mathrm{ppm}$ OTC in distilled water was used. The absorbance of a pure cocktail solution was read for 4 minutes then OTC and buffer were added to the cocktail and the absorbance was read for another 4 minutes. The procedure was repeated 6 times and computed its $\Delta$ absorbance in each trial. Using the computed $\Delta$ absorbance, the relative standard deviation for the 6 trials were obtained. The calculated value of the relative standard deviation will prove whether the method was repeatable or not.

The study is based on the work of Fahelelbom, et. al [5]. The method may not be better than the standard methods due to the substitution, but the resulting figures are within acceptable limits.

\subsection{Sensitivity}

To test the sensitivity of the sensor, serially diluted concentrations from $5 \mathrm{ppm}$ to $1 \mathrm{ppm}$ were tested by measuring the absorbance in each cycle with different concentration using the same working wavelength in a span of 8 minutes per cycle, 4 minutes for cocktail and another 4 minutes for cocktail with OTC and buffer. The absorbance vs time (s) was plotted to compare the difference of the absorbance as the concentration decreases. Also, the $\Delta$ absorbance between cocktail and cocktail with OTC in each cycle were calculated to determine the relationship of $\Delta$ absorbance and concentration of OTC (ppm).

The relationship of $\Delta$ absorbance and concentration of OTC (ppm) were analyzed by plotting $\Delta$ absorbance vs. concentration of OTC (ppm) to further determine the linearity of $\triangle$ absorbance and concentration of OTC (ppm).

This procedure was repeated three times to ensure the accuracy of the data obtained. The calibration curve and linearity were obtained.

\subsection{Limit of detection}

The limit of detection of the method used was computed using the equation:

$$
L O D=\frac{3 S D}{m}
$$

where SD is the standard deviation obtained from the stability test done at the start of the experiment, and $\mathrm{m}$ is the slope of the correlation equation produced from the sensitivity analysis of the samples tested.

\subsection{Real water sample}

Real freshwater samples were gathered from the shrimp hatchery at Brgy. Sindol, San Felipe, Zambales and tested based from the new method used in order to determine the concentration of OTC in the samples. The freshwater samples from the hatchery were already treated and contained approximately $3 \mathrm{ppm}$ of OTC prior to the gathering of the sample. Three trials were made, and the resulting absorbance readings were checked for their accuracy using the correlation equation formed from sensitivity analysis.

\section{Results and discussion}

\subsection{Preparation of solutions}

The cocktail solution, freshly prepared for each tests, gave a color of yellowish hue. OTC, in powder form, 
was yellow, but since concentration was just in the range of $5 \mathrm{ppm}$ to $1 \mathrm{ppm}$, it gives a faint color when dissolved in distilled water. The two solutions tested were stable throughout the experiments. However, the cocktail solution reacts with the styrene cuvette resulting into a foggy cuvette, therefore, glass cuvettes were used for the experiments done.

\subsection{Determination of working wavelength}

The results from the determination of working wavelength were plotted as absorbance vs. wavelength. Based from the graph shown in the Fig. 1, the optimum difference was found at the wavelength of $519 \mathrm{~nm}$. Therefore, the working wavelength that was used in the proceeding experiments was $519 \mathrm{~nm}$. The decrease in the peak using only water may be due to the wave effect of an $\mathrm{OH}$ entering the rings of the OTC than the $\mathrm{C}-\mathrm{OH}$ effect.

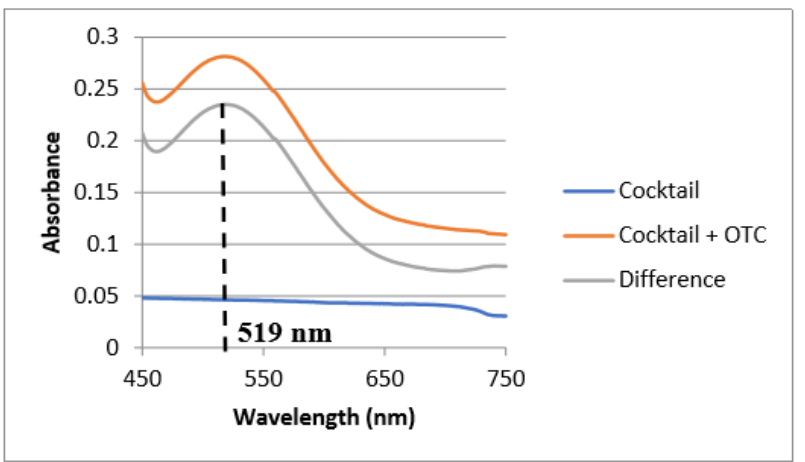

Fig. 1. Spectra of the OTC with the cocktail

\subsection{Signal stability test}

The stability of the cocktail solution was tested using UV-Vis Spectrophotometer at the working wavelength of $519 \mathrm{~nm}$ for exactly 1 hour. The results were plotted as Absorbance vs Time (s) as shown in the Fig. 2.

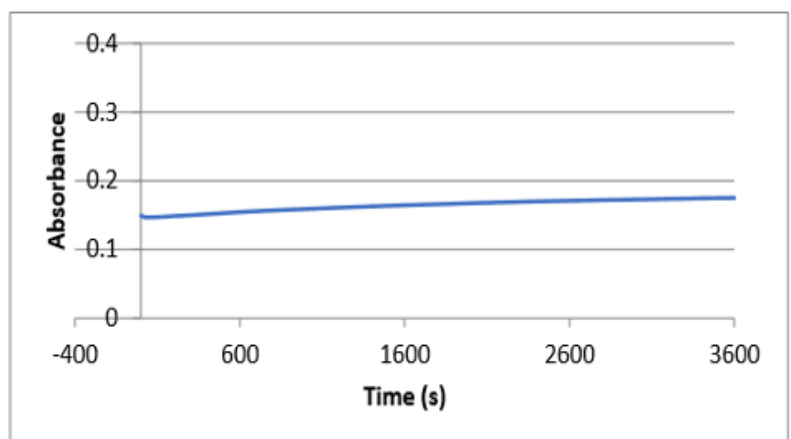

Fig. 2. Stability. Graph of Absorbance vs. Time (s)

The stability test gave a relative standard deviation of $5.07 \%$, which was below $10 \%$ limit. This just proved that the signal used was stable.

The $10 \%$ limit used in the experiments done was based on previous studies conducted which used the $10 \%$ limit as a measure of the reliability of the results produced. Additionally, the acceptable RSD value for sensors is commonly less than $10 \%$ [5].

\subsection{Repeatability}

The method was tested of its repeatability using UV-Vis Spectrophotometer at the wavelength of $519 \mathrm{~nm}$. The results were plotted as Absorbance vs Time (s). As shown in Fig. 3, 6 trials were made using 3 ppm OTC in distilled water sample.

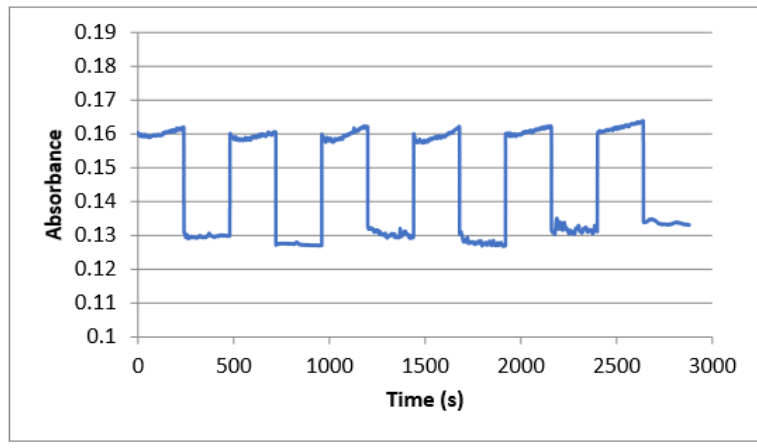

Fig. 3. Repeatability. Graph of Absorbance vs. Time (s) $(n=6)$

The method has proven repeatable giving an RSD of $5.00 \%$, which was below $10 \%$ limit.

\subsection{Sensitivity}

The sensitivity was tested using different concentrations of OTC which was serially diluted from a concentration of $5 \mathrm{ppm}$ to $1 \mathrm{ppm}$. Shown in figure 4 below is the sensitivity summary of the 3 trials made. Plot of $\triangle$ Absorbance vs OTC concentration (ppm) shows the relationship of $\Delta$ absorbance with OTC concentration, as the concentration increases the $\Delta$ absorbance also increases. The figure 4 shows the linear relationship of $\Delta$ absorbance with OTC concentration and showed an $\mathrm{R}^{2}$ value of 0.9892 .

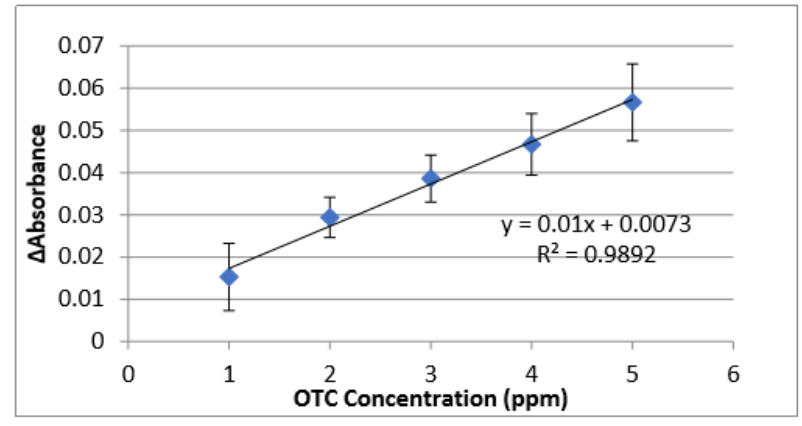

Fig. 4. Sensitivity Test ( $\mathrm{n}=3)$. $\Delta$ Absorbance vs. Concentration (ppm)

\subsection{Limit of detection}

Using the previously defined equation for the determination of the limit of detection of the sample, the computed LOD based from the data gathered is:

$$
L O D=\frac{3 S D}{m}=\frac{3 * 0.001787}{0.01}=0.5
$$


The computed LOD was based on the lower limit of $1 \mathrm{ppm}$ concentration of OTC in water, respectively. The limit of detection gives an allowance as to the lowest concentration readings that could be detected by the method used. In this case, the lowest concentration that can be read is until $0.46 \mathrm{ppm}$ of OTC.

\subsection{The method for real water sample testing}

Fig. 5 shows the plot of absorbance against time for the real freshwater sample with an unknown OTC concentration. The absorbance readings of the pure cocktail solution were consistent to be around $0.16-$ 0.18 , and when the real water sample was added to the cocktail liquid, the solution formed was found to have absorbance readings of around $0.21-0.22$, slightly higher than the pure cocktail solution. The absolute difference in absorbance between the cocktail solution and the cocktail solution with water sample is around $0.04-0.05$. Substituting the equation obtained in the signal stability test, $\mathrm{y}=0.01 \mathrm{x}+0.0073$, where $\mathrm{x}$ and $\mathrm{y}$ are concentration and change in absorbance respectively, the OTC concentration of the real sample was around 3.2 $-4.2 \mathrm{ppm}$.

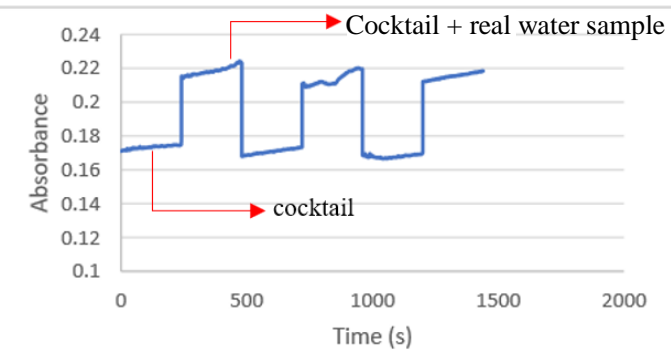

Fig. 5. Real water repeatability test. Absorbance vs. Time (s) Graph $(n=3)$

A relative standard deviation value of $2.46 \%$ was obtained, indicating that the readings obtained from the three consecutive trials were consistent.

However, it could be worthy to consider the response of the real water sample. Instead of the usual decrease in absorbance as observed from the laboratory standard samples, an increase in absorbance was observed for the real water sample, which may have been caused by the presence of a other substances aside from OTC in the sample, or the material present may have overcome the effect of OTC in the freshwater sample. With these results, another wavelength scan was done in order to verify if the working wavelength for the laboratory standard samples is the same as that of the real water samples. Fig. 6 shows the spectra of the real water sample with and without the cocktail solution.

From the plot of absorbance against wavelength for the real water sample, the original working wavelength of $519 \mathrm{~nm}$ became one of the lowest points in the graph. This shift in the absorbance reading for the real water sample may have been caused by the presence of interfering substances in the real water sample.
Additionally, the presence of another antibiotic in the freshwater sample tested, chloramphenicol, also contributed to the sudden change in the absorbance reading for the real water sample.

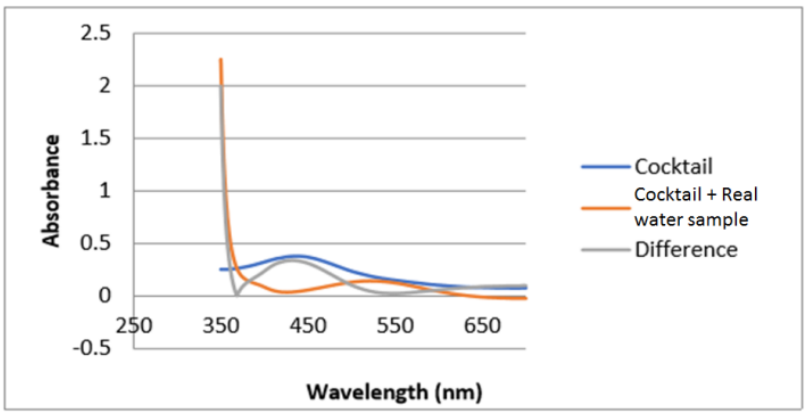

Fig. 6. Spectra of Real Water Sample

\subsection{Optical sensor features}

Based on the wavelength scan, stability, repeatability, and sensitivity experiments done on the samples, including the calculated limit of detection, Table $\mathbf{1}$ presents a summary of the features of the optical sensor produced. The results obtained for the sensor produced is also compared with the results of a previous study done, using methanol as the OTC solvent.

Table 1. Optical sensor features

\begin{tabular}{c|c|c}
\hline TEST & $\begin{array}{c}\text { PROPOSED } \\
\text { METHOD }\end{array}$ & $\begin{array}{c}\text { FAHELELBOM, } \\
\mathbf{2 0 0 8}\end{array}$ \\
\hline OTC Solvent & Water & Methanol \\
\hline Wavelength & $519 \mathrm{~nm}$ & $540 \mathrm{~nm}$ \\
\hline Stability & $\mathrm{RSD}=5.07 \%$ & $\mathrm{RSD}=1.28 \%$ \\
\hline Repeatability & $\mathrm{RSD}=5.00 \%$ & $\mathrm{RSD}=1.20 \%$ \\
\hline Sensitivity & $0.01 \mathrm{abs} / \mathrm{ppm}$ & $0.0433 \mathrm{abs} / \mathrm{ppm}$ \\
\hline Linearity & $\mathrm{R}^{2}=0.9892$ & $\mathrm{R}^{2}=0.9989$ \\
\hline $\begin{array}{c}\text { Limit of } \\
\text { Detection }\end{array}$ & $0.54 \mathrm{ppm}$ & $0.5 \mathrm{ppm}$ \\
\hline $\begin{array}{c}\text { Concentration } \\
\text { range }\end{array}$ & $1.0-5.0 \mathrm{ppm}$ & $5.0-40.0 \mathrm{ppm}$ \\
\hline
\end{tabular}

\section{Conclusion and recommendation}

\subsection{Conclusion}

The accuracy and reliability of the testing method used was measured using the computed RSD values for the different figures of merit. The stability, repeatability, sensitivity, and limits of detection of the method were obtained, and RSD values of less than $10 \%$ for each of the parameters are achieved. The RSD values obtained for each of the figures of merit were found to be $5.07 \%$ for stability, and $5.00 \%$ for repeatability of the $3 \mathrm{ppm}$ sample. A wavelength scan was performed on the cocktail liquid, consisting of both chloranilic acid acetonitrile solution and pure acetonitrile, together with oxytetracycline (the antibiotic) and $\mathrm{pH} 9$ buffer, in order to determine the wavelength at which the difference between the absorbance of the two solutions tested was highest. Maximum absorbance difference for the 
wavelength scan was observed at $519 \mathrm{~nm}$. A correlation equation of $\mathrm{y}=0.01 \mathrm{x}+0.0073$, with an $\mathrm{R}^{2}$ reading of 0.9892 from the initial concentration of $1 \mathrm{ppm}$ to the final concentration of $5 \mathrm{ppm}$, was obtained from the sensitivity analysis, indicating that the trend is linear. Using the slope value of 0.01 , and the resulting standard deviation value from the stability analysis, the limit of detection was calculated to be 0.5 , demonstrating that the upper and lower limits of the concentration detected by the method are $5.5 \mathrm{ppm}$ and $0.45 \mathrm{ppm}$, respectively. Real water sample were also tested for repeatability, and an RSD value of $2.455 \%$ was achieved. It was also found out that the concentration of OTC on the freshwater sample was at 3-4 ppm and did not exceed the limit of 5 ppm, indicating that the absorbance reading demonstrated by the real water sample is within the limits of detection of the method.

The testing method produced was proved to be efficient as it could produce results only within a few minutes. During the testing of the real water sample, it only took a few minutes before the concentration of Oxytetracycline residue was determined with the use of the correlation equation formed from the sensitivity analysis, using the absorbance reading of the sample as the determining factor.

\subsection{Recommendation}

It is then recommended that future studies be done to produce and materialize the method for ease of determination of Oxytetracycline residue concentration in freshwater ponds in the aquaculture industry. It is also recommended to perform the response time to have an additional support to the claim of method efficiency. Additionally, it is recommended that interference studies be conducted.

The authors would like to express their deepest gratitude to the University of Santo Tomas Chemical Engineering Department, Faculty of Engineering, and to the Research Center for Natural and Applied Sciences for allowing us to work in the laboratory and use their equipments.

\section{References}

[1] Leal, J. F., Esteves, V. I., \& Santos, E. B. H. (2016). Use of sunlight to degrade oxytetracycline in marine aquaculture's waters. Environmental Pollution, 213, 932-939. https://doi.org/10.1016/j.envpol.2016.03.040

[2] National Aquaculture Sector Overview (Philippines). (2018). Retrieved from Food and Agriculture Organization of the United Nations: http://www.fao.org/fishery/countrysector/naso_phili ppines/en\#tcN70085

[3] Santos, L., \& Ramos, F. (2016). Analytical strategies for the detection and quantification of antibiotic residues in aquaculture fishes: A review. Trends in Food Science and Technology, 52, 16-30. https://doi.org/10.1016/j.tifs.2016.03.015
[4] Tlili, I. e. (2016). Simultaneous detection of antibiotic and other drug residues in the dissolved and particulate phases of water by an off-line SPE combined with on-line SPE-LC-MS/MS: Method development and application. Science of the Total Environment, 425.

[5] Fahelelbom, K.M.S. (2008). Analysis of Certain Tetracyclines and Oxytetracyclines through Charge Transfer Complexation Khairi M . S . Fahelelbom College of Pharmacy, AL-Ain University of Science and Technology, Al-Ain 64141, United Arab Emirates. American Journal of Pharmacology and Toxicology, 3(3), 212-218. 